\title{
Scalar-tensor teleparallel wormholes by Noether symmetries
}

\author{
Sebastian Bahamonde, ${ }^{1,{ }^{*}}$ Ugur Camci, ${ }^{2, \dagger}$ Salvatore Capozziello, ${ }^{3,4,5,6, \sharp}$ and Mubasher Jamil ${ }^{7, \S}$ \\ ${ }^{1}$ Department of Mathematics, University College London, Gower Street, \\ London WCIE 6BT, United Kingdom \\ ${ }^{2}$ Department of Physics, Faculty of Sciences, Akdeniz University, 07058 Antalya, Turkey \\ ${ }^{3}$ Dipartimento di Fisica, Universitá di Napoli Federico II, I-80126 Napoli, Italy \\ ${ }^{4}$ INFN Sez. di Napoli, Compl. Univ. di Monte S. Angelo, Edificio G, Via Cinthia, I-80126 Napoli, Italy \\ ${ }^{5}$ Gran Sasso Science Institute (INFN), Via F. Crispi 7, I-67100 L' Aquila, Italy \\ ${ }^{6}$ Tomsk State Pedagogical University, ul. Kievskaya, 60, 634061 Tomsk, Russia \\ ${ }^{7}$ Department of Mathematics, School of Natural Sciences (SNS), National University of Sciences \\ and Technology (NUST), H-12 Islamabad, Pakistan
}

(Received 12 August 2016; revised manuscript received 19 September 2016; published 26 October 2016)

A gravitational theory of a scalar field nonminimally coupled with torsion and a boundary term is considered with the aim to construct Lorentzian wormholes. Geometrical parameters including shape and redshift functions are obtained for these solutions. We adopt the formalism of the Noether gauge symmetry approach in order to find symmetries, Lie brackets and invariants (conserved quantities). Furthermore by imposing specific forms of potential function, we are able to calculate metric coefficients and discuss their geometrical behavior.

DOI: $10.1103 /$ PhysRevD.94.084042

\section{INTRODUCTION}

The notion of Lorentzian wormholes (WH) arose when Morris and Thorne explored the possibility of time travel for humans using the principles of general relativity (GR) [1]. Einstein's theory of GR predicts that the structure and geometry of spacetime in the presence of matter is not rigid but is elastic and deformable. The more compact the object is, the more strong the curvature of space is, which essentially leads to the idea of black holes. However in the latter case, the fabric of spacetime loses its meaning at the curvature singularity. If somehow the formation of singularity is avoided then it would be possible to travel in and out of the horizon.

The possibility of such a solution to the Einstein field equations was explored for the first time by Flamm [2] soon after the formulation of GR, but it was later shown that his solution was unstable. A typical wormhole is a tubelike structure which is asymptotically flat from both sides. The radius of the wormhole throat could be constant or variable depending on its construction and it is termed static or nonstatic, respectively. GR predicts that to form a $\mathrm{WH}$, an exotic form of matter (violating the energy conditions) must be present near the throat of the WH. The problem is the dearth of reasonable sources sustaining the wormhole geometry. One possible candidate is the phantom energy (which is a cosmic dynamical scalar field with negative kinetic energy in its Lagrangian) and it is one of the

\footnotetext{
sebastian.beltran.14@ucl.ac.uk

tucamci@akdeniz.edu.tr

capozziello@na.infn.it

§mjamil@sns.nust.edu.pk
}

candidates for explaining cosmic accelerated expansion as well [3]. Since the existence of phantom energy is questionable and no other suitable exotic matter candidate is available at the moment, an alternative approach is commonly followed: investigation if the modifications of laws of gravity (i.e. GR), proposed primarily for explanation of accelerated expansion and avoiding singularities, can support the WH geometries. Since the WH is a nonvacuum solution of Einstein field equations, the presence of some form of energy matter is necessary to construct a WH. In the framework of modified gravity, the matter content is assumed to satisfy the energy conditions near the WH throat, while higher curvature correction terms in the Lagrangian are required to sustain the WH geometry.

Like $f(R)$ (where $R$ is a Ricci scalar) gravity which is based on a symmetric connection, the $f(T)$ (where $T$ is torsion of spacetime) gravity is based on a skew-symmetric connection [4]. The latter theory is one of the many alternative (or modified) theories of gravity available in the literature [5-8]. $f(T)$ theory is based on the tetrad formalism and the governing equations are derived by varying the gravitational Lagrangian with respect to the tetrads. Since the tetrad is not unique, the governing equations acquire different forms and hence different solutions in different tetrads (see details in Sec. II). Like any other theory, $f(T)$ theory has several drawbacks: it does not obey local Lorentz invariance, it violates the first law of thermodynamics, and it does not have unique governing field equations [9]. Despite these problems, the theory efficiently describes the cosmic accelerated expansion and predicts the existence of new kinds of black holes and wormholes. It can also resolve the dark energy 
and dark matter conundrums [10]. Recently some attempts have been made to unify both $f(R)$ and $f(T)$ theories as $f(R, T)$ gravity by introducing a boundary term $B$ in $f(T, B)$ gravity [11-13].

Here, we want to study wormholes in a theory where torsion is nonminimally coupled with a scalar field and includes a matter action. It is important to note that similar models have also been studied in the literature [14].

In this paper, we employ the Noether symmetry approach [15] and a wormhole metric ansatz to obtain the governing system of differential equations. After solving the equations, we get the form of metric coefficients, symmetry generators, invariants and the form of torsion and potential functions. This approach has been extensively studied in the literature [16-20].

The plan of the paper is as follows: In Sec. II, we provide a brief review of generalized teleparallel gravity and sketch relevant notations. In Sec. III, we present the model starting with the action of a nonminimally coupled scalar field with both torsion and the boundary term. We also derive the field equations and choose an ansatz for the wormhole metric. In Sec. IV and its subsections, we construct a pointlike Lagrangian, search for Noether symmetries and derive the wormhole solutions. Discussion and conclusions are given in Sec. V.

\section{TELEPARALLEL EQUIVALENT OF GENERAL RELATIVITY}

In what follows we follow conventions outlined in [21]. Here we present a brief review of the teleparallel formalism of GR. Unlike GR, the dynamical variable in the teleparallel theory is the tetrad $e_{\mu}^{a}$ (rather than the metric tensor), where greek and latin indices denote spacetime and tangent space indices, respectively. Here the metric tensor $g_{\mu \nu}$ is related to the tetrads as

$$
g_{\mu \nu}=e_{\mu}^{a} e_{\nu}^{b} \eta_{a b},
$$

where $\eta_{a b}$ denotes the Minkowski metric tensor. The inverse tetrad $E_{a}^{\mu}$ is defined as

$$
E_{m}^{\mu} e_{\mu}^{n}=\delta_{m}^{n}, \quad \text { and } \quad E_{m}^{\nu} e_{\mu}^{m}=\delta_{\mu}^{\nu} .
$$

Here $e$ is the determinant of the tetrad $e_{\mu}^{a}$, which can be evaluated from the determinant of the metric tensor $e=\sqrt{-g}$.

GR is based on the symmetric Levi-Civita connection, whereas teleparallel gravity relies on the antisymmetric Weitzenböck connection $W_{\mu}{ }^{a}{ }_{\nu}$ defined as

$$
W_{\mu}^{a}{ }_{\nu}=\partial_{\mu} e^{a}{ }_{\nu} .
$$

The antisymmetric nature of connection leads to the concept of torsion in geometry. The torsion tensor is the antisymmetric part of the Weitzenböck connection,

$$
T_{\mu \nu}^{a}=W_{\mu}^{a}{ }_{\nu}-W_{\nu}{ }^{a}{ }_{\mu}=\partial_{\mu} e_{\nu}^{a}-\partial_{\nu} e_{\mu}^{a} .
$$

The tensor $T_{\mu}$, referred to as the torsion vector, is defined through the contraction of the torsion tensor, i.e.

$$
T_{\mu}=T_{\lambda \mu}^{\lambda} .
$$

To construct the field equations of teleparallel gravity, we consider the following Lagrangian density and vary it with respect to the tetrad,

$$
\mathcal{L}_{T}=\frac{e}{2 \kappa^{2}} S^{a b c} T_{a b c}
$$

where

$S^{a b c}=\frac{1}{4}\left(T^{a b c}-T^{b a c}-T^{c a b}\right)+\frac{1}{2}\left(\eta^{a c} T^{b}-\eta^{a b} T^{c}\right)$.

The torsion scalar $T$ is defined as

$$
T=S^{a b c} T_{a b c} .
$$

Furthermore, to express teleparallel gravity as an equivalent of GR, we write the Levi-Civita connection ${ }^{0} \Gamma$ in terms of the Weitzenböck connection as

$$
{ }^{0} \Gamma_{\lambda \rho}^{\mu}=W_{\lambda^{\mu}}{ }_{\rho}-K_{\lambda^{\mu}}{ }_{\rho},
$$

where here $K$ is called the contortion tensor and it is defined as

$$
2 K_{\mu}^{\lambda}{ }_{\nu}=T_{\mu \nu}^{\lambda}-T_{\nu \mu}^{\lambda}+T_{\mu}^{\lambda}{ }_{\nu}^{\lambda} .
$$

This contortion tensor is antisymmetric in its last two indices. Now expressing the Ricci scalar of the Levi-Civita connection in terms of the Weitzenböck connection, using (9), we get

$$
R=-T+\frac{2}{e} \partial_{\mu}\left(e T^{\mu}\right)
$$

As the difference between the Ricci scalar and the torsion scalar is simply a total derivative, the action (6) gives rise to the same dynamics as the Einstein Hilbert action. This shows that teleparallel gravity is indeed equivalent to GR. Defining the boundary quantity

$$
B=\frac{2}{e} \partial_{\mu}\left(e T^{\mu}\right)
$$

one then has simply the relation $R=-T+B$. Note that one can write $B$ in terms of a Levi-Civita covariant derivative simply as $B=2 \nabla_{\mu} T^{\mu}$. 


\section{NONMINIMALLY COUPLED SCALAR FIELD TO TORSION AND BOUNDARY TERM}

Let us consider the following gravitational action which describes a nonminimally coupled scalar field to both torsion and the boundary term $[22,23]$,

$S=\int\left[\frac{1}{2}\left(f(\phi) T+g(\phi) B+\partial_{\mu} \phi \partial^{\mu} \phi\right)-V(\phi)\right] e d^{4} x$.

Here, $V(\phi)$ is the scalar field potential and $f(\phi)$ and $g(\phi)$ are smooth functions of the scalar field $\phi$. This action is not a new theory; instead it is a generalization or rather a unification of different theories into one action. This theory is very rich in the sense that one can recover very wellknown scalar-tensor theories. In fact, let us for example choose

$$
f(\phi)=1-\xi \phi^{2}, \quad g(\phi)=-\chi \phi^{2},
$$

where $\xi$ and $\chi$ are coupling constants. Depending on the value of $\chi$ and $\xi$, we can recover scalar-tensor theories nonminimally coupled with the torsion scalar $(\chi=0)$, with the boundary term $(\xi=0)$, the Ricci scalar $(\chi=-\xi)$ and also quintessence theory $(\xi=\chi=0)$. The latter theories have been widely studied in the literature from cosmology to astrophysical sources like wormholes. Traversable wormholes supported by a nonminimally coupled scalar field with the Ricci scalar $(\chi=-\xi)$ were first studied in
Ref. [24]. The authors found that depending on the value of the coupling constant, one can construct different kinds of wormholes. Wormholes supported by a kinklike configuration of a scalar field were studied in [25]. Moreover, in $[26,27]$, the authors studied the stability of electrically charged and neutral wormholes within this theory.

Additionally, Brans-Dicke scalar-tensor theory with $w(\phi)=1$ can be recovered if we set

$$
f(\phi)=\xi \phi^{2}, \quad g(\phi)=-\chi \phi^{2}
$$

with $\chi=-\xi$. Wormhole configurations under this theory have been widely studied in the literature [28]. One interesting feature of Bans-Dicke wormholes is that they can satisfy the energy conditions. For the reader interested in cosmology in all of these theories mentioned above, see Refs. [22,29,30]. In the context of teleparallel gravity, wormhole solutions have also been studied (see [31] and referees therein), but to the best of our knowledge, wormholes in teleparallel scalar-tensor theories (for example with $\xi \neq-\chi)$ have not been considered until now. Note that action is very rich since we can study all these kinds of theories and then make the corresponding choice of the coupling functions. Moreover, using this approach, we can have a direct relation from the teleparallel and metric counterparts.

By varying this action with respect to the tetrad field we find the following field equations:

$$
\begin{aligned}
& 2 f(\phi)\left[e^{-1} \partial_{\mu}\left(e S_{a}{ }^{\mu \nu}\right)-E_{a}^{\lambda} T^{\rho}{ }_{\mu \lambda} S_{\rho}{ }^{\nu \mu}-\frac{1}{4} E_{a}^{\nu} T\right]-E_{a}^{\nu}\left[\frac{1}{2} \partial_{\mu} \phi \partial^{\mu} \phi-V(\phi)\right] \\
& \quad+E_{a}^{\mu} \partial^{\nu} \phi \partial_{\mu} \phi+2\left(\partial_{\mu} f(\phi)+\partial_{\mu} g(\phi)\right) E_{a}^{\rho} S_{\rho}{ }^{\mu \nu}+E_{a}^{\nu} \square g(\phi)-E_{a}^{\mu} \nabla^{\nu} \nabla_{\mu} g(\phi)=0 .
\end{aligned}
$$

By contracting this equation with the tetrad field $e_{\lambda}^{a}$, we can have this equation only in spacetime index

$$
\begin{aligned}
& 2 f(\phi)\left[e^{-1} e_{\lambda}^{a} \partial_{\mu}\left(e S_{a}{ }^{\mu \nu}\right)-T^{\rho}{ }_{\mu \lambda} S_{\rho}{ }^{\mu \mu}-\frac{1}{4} \delta_{\lambda}^{\nu} T\right]-\delta_{\lambda}^{\nu}\left[\frac{1}{2} \partial_{\mu} \phi \partial^{\mu} \phi-V(\phi)\right] \\
& \quad+\partial^{\nu} \phi \partial_{\lambda} \phi+2\left(\partial_{\mu} f(\phi)+\partial_{\mu} g(\phi)\right) S_{\lambda}{ }^{\mu \nu}+\delta_{\lambda}^{\nu} \square g(\phi)-\nabla^{\nu} \nabla_{\lambda} g(\phi)=0 .
\end{aligned}
$$

Now, by varying the action (13) with respect to the scalar field we obtain the so-called Klein-Gordon equation which reads

$$
\square \phi+V^{\prime}(\phi)=\frac{1}{2}\left(f^{\prime}(\phi) T+g^{\prime}(\phi) B\right) .
$$

Let us consider a static spherically symmetric spacetime in which the metric is

$$
d s^{2}=e^{a(r)} d t^{2}-e^{b(r)} d r^{2}-M(r)^{2} d \Omega^{2},
$$

where $a(r), b(r)$ and $M(r)$ are functions of the radial coordinate $r$, and $d \Omega^{2}=d \theta^{2}+\sin ^{2} \theta d \varphi^{2}$. The corresponding off-diagonal tetrad field to the metric (19) is 


$$
e_{\mu}^{a}=\left(\begin{array}{cccc}
e^{a / 2} & 0 & 0 & 0 \\
0 & e^{b / 2} \sin \theta \cos \varphi & M(r) \cos \theta \cos \varphi & -M(r) \sin \theta \sin \varphi \\
0 & e^{b / 2} \sin \theta \sin \varphi & M(r) \cos \theta \sin \varphi & M(r) \sin \theta \cos \varphi \\
0 & e^{b / 2} \cos \theta & -M(r) \sin \theta & 0
\end{array}\right) .
$$

This tetrad field has been used in the context of $f(T)$ gravity since it does not produce a constraint, namely $f_{T T}=0$, as a diagonal tetrad. For this tetrad, the field equations read

$$
\begin{gathered}
V(\phi)+\frac{1}{2} e^{-b(r)} \phi^{\prime 2}+\frac{e^{-b(r)}}{2 r}\left(r b^{\prime}(r) g^{\prime}(\phi)+4 f^{\prime}\right)-\frac{f(\phi)}{r}\left(e^{-b(r)}\left(b^{\prime}(r)-\frac{1}{r}\right)+\frac{1}{r}\right)-e^{-b(r)} g^{\prime \prime}(\phi) \\
-\frac{2 e^{-\frac{b(r)}{2}}}{r} \phi^{\prime}\left(f^{\prime}(\phi)+g^{\prime}(\phi)\right)=0, \\
V(\phi)-\frac{1}{2} e^{-b(r)} \phi^{\prime 2}-\frac{f(\phi)}{r}\left(\frac{1}{r}-e^{-b(r)}\left(a^{\prime}(r)+\frac{1}{r}\right)\right)-e^{-b(r)}\left(\frac{a^{\prime}(r)}{2}+\frac{2}{r}\right) g^{\prime}(\phi)=0, \\
V(\phi)+\frac{1}{2} e^{-b(r)} \phi^{\prime 2}+e^{-b(r)}\left(\frac{a^{\prime}(r)}{2}+\frac{1}{r}\right) f^{\prime}(\phi)-e^{-b(r)}\left(g^{\prime \prime}(\phi)-\frac{1}{2} b^{\prime}(r) g^{\prime}(\phi)\right) \\
+\frac{1}{2} e^{-b(r)} f(\phi)\left(a^{\prime \prime}(r)+\frac{1}{2} a^{\prime}(r)\left(a^{\prime}(r)-b^{\prime}(r)\right)+\frac{a^{\prime}(r)}{r}-\frac{b^{\prime}(r)}{r}\right)-\frac{e^{-\frac{b(r)}{2}}}{r} \phi^{\prime}\left(f^{\prime}(\phi)+g^{\prime}(\phi)\right)=0 .
\end{gathered}
$$

Here, primes denote differentiation with respect to the radial coordinate $r$. It can be shown that if we choose $f(\phi)=$ $1+\xi \phi^{2}=-g(\phi)$ we recover the case studied in [24,25]. Additionally, the Klein-Gordon equation becomes

$$
-e^{-b(r)} \phi^{\prime}(r)\left(\frac{1}{2} a^{\prime}(r)-\frac{b^{\prime}(r)}{2}+\frac{2}{r}\right)-e^{-b(r)} \phi^{\prime \prime}+V^{\prime}(\phi)-\frac{1}{2}\left(B(r) g^{\prime}(\phi)+T(r) f^{\prime}(\phi)\right)=0,
$$

where the torsion scalar and the boundary term are respectively given by

$$
\begin{gathered}
T(r)=\frac{2 e^{-b(r)}}{M^{2}}\left(e^{\frac{b(r)}{2}}-M^{\prime}\right)\left(-M a^{\prime}-M^{\prime}+e^{\frac{b(r)}{2}}\right), \\
B(r)=-2 e^{-\frac{b(r)}{2}}\left(\frac{a^{\prime}}{M}+\frac{2 M^{\prime}}{M^{2}}\right)+e^{-b(r)}\left[a^{\prime \prime}-\frac{1}{2} a^{\prime} b^{\prime}+\frac{1}{2} a^{\prime 2}+\frac{4 M^{\prime} a^{\prime}}{M}-\frac{2 M^{\prime} b^{\prime}}{M}+\frac{4 M^{\prime 2}}{M^{2}}+\frac{4 M^{\prime \prime}}{M}\right] .
\end{gathered}
$$

Clearly, by subtracting (26) with (25) we recover the Ricci scalar,

$$
R(r)=-T(r)+B(r)=-\frac{2}{M^{2}}+e^{-b(r)}\left[a^{\prime \prime}-\frac{1}{2} a^{\prime} b^{\prime}+\frac{1}{2} a^{\prime 2}+\frac{2 M^{\prime} a^{\prime}}{M}-\frac{2 M^{\prime} b^{\prime}}{M}+\frac{2 M^{\prime 2}}{M^{2}}+\frac{4 M^{\prime \prime}}{M}\right] .
$$

\section{WORMHOLE SOLUTIONS}

Now we have all the ingredients to study wormhole configurations in this framework. A spherically symmetric wormhole is given by choosing

$$
e^{b(r)}=\left(1-\frac{\beta(r)}{r}\right)^{-1}
$$

where $\beta(r)$ is the shape function of the wormhole and $a(r)$ is known as the redshift function. Thus, the field equations read 


$$
\begin{gathered}
V(\phi)+\left(1-\frac{\beta(r)}{r}\right)\left(\frac{1}{2} \phi^{\prime 2}+\frac{2}{r} f^{\prime}(\phi)-g^{\prime \prime}(\phi)\right)-\frac{\beta^{\prime}(r)}{r^{2}} f(\phi)+\left(\beta^{\prime}(r)-\frac{\beta(r)}{r}\right) \frac{g^{\prime}(\phi)}{2 r} \\
-\frac{2\left(f^{\prime}(\phi)+g^{\prime}(\phi)\right)}{r} \sqrt{1-\frac{\beta(r)}{r}}=0, \\
V(\phi)-\frac{1}{2} \phi^{\prime 2}-\left(\beta(r) a^{\prime}(r)-r a^{\prime}(r)+\frac{\beta(r)}{r}\right) \frac{f(\phi)}{r^{2}}-\left(\frac{a^{\prime}(r)}{2}-\frac{\beta(r) a^{\prime}(r)}{2 r}-\frac{2 \beta(r)}{r^{2}}+\frac{2}{r}\right) g^{\prime}(\phi)+\frac{\beta(r)}{2 r} \phi^{\prime}(r)^{2}=0, \\
V(\phi)+\left(1-\frac{\beta(r)}{r}\right)\left(\frac{1}{2} a^{\prime \prime}(r) f(\phi)+\frac{1}{2} a^{\prime}(r) f^{\prime}(\phi)+\frac{f^{\prime}(\phi)}{r}+\frac{1}{2} \phi^{\prime 2}\right)-g^{\prime \prime}(\phi)+\frac{\left(r a^{\prime}(r)+2\right)}{4 r} a^{\prime}(r) f(\phi) \\
-\left(\frac{1}{2} a^{\prime}(r)^{2}+\frac{a^{\prime}(r)}{2 r}-\frac{1}{r^{2}}\right) \frac{\beta(r) f(\phi)}{2 r}-\left(\frac{a^{\prime}(r)}{2}+\frac{1}{r}\right) \frac{f(\phi) \beta^{\prime}(r)}{2 r}+\frac{\beta^{\prime}(r) g^{\prime}(\phi)}{2 r} \\
-\left(\frac{g^{\prime}(\phi)}{2 r}-g^{\prime \prime}(\phi)\right) \frac{\beta(r)}{r}-\frac{\left(f^{\prime}(\phi)+g^{\prime}(\phi)\right)}{r} \sqrt{1-\frac{\beta(r)}{r}}=0 .
\end{gathered}
$$

In the following section, we use the Noether symmetry approach to find analytical wormhole solutions within this theory.

\section{A. The pointlike Lagrangian}

The action (13) can be written as $S=\int \mathcal{L} d r$, where $\mathcal{L}$ is the Lagrangian density. In the background of the static spherically symmetric spacetime (19), the pointlike Lagrangian density for the teleparallel theory of gravity is obtained as

$$
\begin{aligned}
\mathcal{L}= & f(\phi) e^{\frac{a-b}{2}}\left(e^{b / 2}-M^{\prime}\right)\left[e^{b / 2}-M a^{\prime}-M^{\prime}\right]-g(\phi) e^{a / 2}\left(M a^{\prime}+2 M^{\prime}\right) \\
& -g_{, \phi} e^{\frac{a-b}{2}}\left(\frac{M^{2}}{2} a^{\prime} \phi^{\prime}+2 M M^{\prime} \phi^{\prime}\right)-\frac{M^{2}}{2} e^{\frac{a-b}{2}} \phi^{\prime 2}-M^{2} e^{\frac{a+b}{2}} V(\phi),
\end{aligned}
$$

where $g_{, \phi}=d g(\phi) / d \phi$. Note that the Hessian determinant of the above Lagrangian is 0 . This is clearly due to the absence of the generalized velocity $b^{\prime}$ in the pointlike Lagrangian. By varying the pointlike Lagrangian density (32) with respect to the redshift function $a$, we get

$$
f(\phi)\left[\frac{2 M^{\prime \prime}}{M}+\frac{M^{\prime 2}}{M^{2}}-\frac{b^{\prime} M^{\prime}}{M}-\frac{e^{b}}{M^{2}}\right]+2 f_{, \phi} \frac{\phi^{\prime}}{M}\left(M^{\prime}-e^{b / 2}\right)-g_{, \phi}\left(\phi^{\prime \prime}+2 e^{b / 2} \frac{\phi^{\prime}}{M}\right)+\frac{1}{2} \phi^{2}+e^{b} V_{, \phi}=0 .
$$

Variations with respect to the shape function $b$ give us

$$
f(\phi)\left[\frac{M^{\prime}}{M}\left(a^{\prime}+\frac{M^{\prime}}{M}\right)-\frac{e^{b}}{M^{2}}\right]-g_{, \phi}\left(\frac{a^{\prime} \phi^{\prime}}{2}+\frac{2 M^{\prime} \phi^{\prime}}{M}\right)-\frac{1}{2} \phi^{2}+e^{b} V(\phi)=0 .
$$

Now, if we vary the pointlike Lagrangian density with respect to $M$, we find

$$
\begin{gathered}
f(\phi)\left[\frac{M^{\prime \prime}}{M}+\frac{a^{\prime \prime}}{2}+\left(a^{\prime}-b^{\prime}\right)\left(\frac{a^{\prime}}{4}+\frac{M^{\prime}}{2 M}\right)\right]+f_{, \phi} \phi^{\prime}\left[\frac{a^{\prime}}{2}+\frac{1}{M}\left(M^{\prime}-e^{b / 2}\right)\right] \\
+g_{, \phi}\left[\phi^{\prime}\left(\frac{b^{\prime}}{2}-\frac{e^{b / 2}}{M}\right)-\phi^{\prime \prime}\right]+\left(\frac{1}{2}-g_{, \phi \phi}\right) \phi^{\prime 2}+e^{b} V_{, \phi}=0 .
\end{gathered}
$$

Finally, varying the pointlike Lagrangian density with respect to $\phi$ yields 


$$
\frac{e^{b}}{2}\left[f_{, \phi} T(r)+g_{, \phi} B(r)\right]+\phi^{\prime \prime}+\phi^{\prime}\left[\frac{1}{2}\left(a^{\prime}-b^{\prime}\right)+\frac{2 M^{\prime}}{M}\right]-e^{b} V_{, \phi}=0 .
$$

The latter equation is the Klein-Gordon equation. The energy function associated with $\mathcal{L}$ is defined by

$$
E_{\mathcal{L}}=q^{\prime k} \frac{\partial \mathcal{L}}{\partial q^{\prime k}}-\mathcal{L}
$$

which is also the Hamiltonian of the system. Here, $q^{i}, i=1,2,3,4$, are the generalized coordinates where $q^{i}=\{a, b, M, \phi\}$, for the Lagrangian density (32) of the teleparallel theory of gravity. Then $E_{\mathcal{L}}$ has the following form,

$$
E_{\mathcal{L}}=M^{2} e^{(a-b) / 2}\left[f(\phi)\left(\frac{M^{\prime 2}}{M^{2}}+\frac{a^{\prime} M^{\prime}}{M}-\frac{e^{b}}{M^{2}}\right)-g_{, \phi}\left(\frac{1}{2} a^{\prime} \phi^{\prime}+\frac{2 M^{\prime} \phi^{\prime}}{M}\right)-\frac{1}{2} \phi^{\prime 2}+e^{b} V(\phi)\right],
$$

which vanishes because of Eq. (34) due to the variation with respect to $b$, i.e. $E_{\mathcal{L}}=0$. This can be explicitly solved in terms of $b$ as a function of the remaining generalized coordinates,

$$
e^{b}=\frac{M^{2}\left[-f(\phi)\left(\frac{M^{\prime 2}}{M^{2}}+\frac{a^{\prime} M^{\prime}}{M}\right)+g_{, \phi}\left(\frac{1}{2} a^{\prime} \phi^{\prime}+\frac{2 M^{\prime} \phi^{\prime}}{M}\right)+\frac{1}{2} \phi^{2}\right]}{M^{2} V(\phi)-f(\phi)} .
$$

Then, using (28), the shape function $\beta(r)$ of the wormhole takes the form

$$
\beta(r)=\frac{r\left[-f(\phi)\left(\frac{M^{\prime 2}}{M^{2}}+\frac{a^{\prime} M^{\prime}}{M}-\frac{1}{M^{2}}\right)+g_{, \phi}\left(\frac{1}{2} a^{\prime} \phi^{\prime}+\frac{2 M^{\prime} \phi^{\prime}}{M}\right)+\frac{1}{2} \phi^{\prime 2}+V(\phi)\right]}{\left[-f(\phi)\left(\frac{M^{\prime 2}}{M^{2}}+\frac{a^{\prime} M^{\prime}}{M}\right)+g_{, \phi}\left(\frac{1}{2} a^{\prime} \phi^{\prime}+\frac{2 M^{\prime} \phi^{\prime}}{M}\right)+\frac{1}{2} \phi^{\prime 2}\right]} .
$$

In its present form, $\beta$ is expressed in terms of several arbitrary functions. Below, we determine explicitly $\beta$ as a function of the radial coordinate and qualitatively investigate its behavior.

\section{B. The Noether symmetry approach}

Let us consider a Noether symmetry vector generator [15],

$$
\mathbf{X}=\xi \frac{\partial}{\partial r}+\eta^{i} \frac{\partial}{\partial q^{i}}
$$

where $q^{i}=\{a, b, M, \phi\}$ are the generalized coordinates in the configuration space $\mathcal{Q} \equiv\left\{q^{i}, i=1, \ldots, 4\right\}$ of the Lagrangian, whose tangent space is $\mathcal{T} Q \equiv\left\{q^{i}, q^{\prime i}\right\}$. The components $\xi$ and $\eta^{i}$ of the Noether symmetry generator $\mathbf{X}$ are functions of $r$ and $q^{i}$. The existence of a Noether symmetry implies the existence of a vector field $\mathbf{X}$ given in (41) if the Lagrangian $\mathcal{L}\left(r, a, b, M, \phi, a^{\prime}, b^{\prime}, M^{\prime}, \phi^{\prime}\right)$ satisfies

$$
\mathbf{X}^{[1]} \mathcal{L}+\mathcal{L}\left(D_{r} \xi\right)=D_{r} G
$$

where $\mathbf{X}^{[1]}$ is the first prolongation of the generator (41) in such a form,

$$
\mathbf{X}^{[1]}=\mathbf{X}+\eta^{\prime i} \frac{\partial}{\partial q^{\prime i}},
$$

$G\left(r, q^{i}\right)$ is a gauge function, $D_{r}$ is the total derivative operator with respect to $r, D_{r}=\partial / \partial r+q^{i} \partial / \partial q^{i}$, and $\eta^{\prime i}$ is defined as $\eta^{\prime i}=D_{r} \eta^{i}-q^{\prime i} D_{r} \xi$. It is important to give the following Noether first integral to emphasize the significance of Noether symmetry that if $\mathbf{X}$ is the Noether symmetry generator corresponding to the Lagrangian $\mathcal{L}\left(r, q^{i}, q^{\prime i}\right)$, then

$$
I=-\xi E_{\mathcal{L}}+\eta^{i} \frac{\partial \mathcal{L}}{\partial q^{\prime i}}-G
$$

which is also the Hamiltonian or a conserved quantity associated with the generator $\mathbf{X}$. Now we seek the condition in which the Lagrangian density (32) would admit any Noether symmetry. The Noether symmetry condition (42) for the Lagrangian (32) gives rise to the following set of differential equations: 


$$
\begin{aligned}
& \xi_{, a}=0, \quad \xi_{, b}=0, \quad \xi_{, M}=0, \quad \xi_{, \phi}=0, \\
& 2 f \eta_{, a}^{3}-g_{, \phi} M \eta_{, a}^{4}=0, \quad 2 f \eta_{, b}^{3}-g_{, \phi} M \eta_{, b}^{4}=0, \\
& \frac{1}{2} M\left(\eta^{1}-\eta^{2}\right)+2 \eta^{3}+g_{, \phi}\left(M \eta_{, \phi}^{1}+4 \eta_{, \phi}^{3}\right)+M\left(2 \eta_{, \phi}^{4}-\xi_{, r}\right)=0 \\
& f\left(\frac{1}{2} M \eta_{, b}^{1}+\eta_{, b}^{3}\right)-g_{, \phi} M \eta_{, b}^{4}=0, \quad g_{, \phi}\left(\frac{1}{2} M \eta_{, b}^{1}+2 \eta_{, b}^{3}\right)+M \eta_{, b}^{4}=0, \\
& f\left[\frac{1}{2}\left(\eta^{1}-\eta^{2}\right)+M \eta_{, M}^{1}+2 \eta_{, M}^{3}-\xi_{, r}\right]+f_{, \phi} \eta^{4}-2 g_{, \phi} M \eta_{, M}^{4}=0, \\
& g_{, \phi}\left(M \eta_{, a}^{4}+\frac{1}{4} M^{2} \eta_{, M}^{4}\right)-f\left[\frac{1}{4} M\left(\eta^{1}-\eta^{2}\right)+\frac{1}{2} \eta^{3}+\eta_{, a}^{3}+\frac{1}{2} M\left(\eta_{, a}^{1}+\eta_{, M}^{3}\right)\right]-\frac{1}{2} f_{, \phi} \eta^{4}=0, \\
& g_{, \phi}\left[\frac{1}{2} M\left(\eta^{1}-\eta^{2}\right)+2 \eta^{3}+4 \eta_{, a}^{3}+M\left(\eta_{, a}^{1}+\eta_{, \phi}^{4}-\xi_{, r}\right)\right]+g_{, \phi \phi} M \eta^{4}+2 M \eta_{, a}^{4}-2 f \eta_{, \phi}^{3}=0, \\
& g_{, \phi}\left[\frac{1}{2} M\left(\eta^{1}-\eta^{2}\right)+\eta^{3}+\frac{1}{4} M^{2} \eta_{, M}^{1}+M\left(\eta_{, M}^{3}+\eta_{, \phi}^{4}-\xi_{, r}\right)\right]+g_{, \phi \phi} M \eta^{4}-\frac{1}{2} f M \eta_{, \phi}^{1}-f \eta_{, \phi}^{3}+\frac{1}{2} M^{2} \eta_{, M}^{4}=0 \\
& (f+g)\left[\frac{1}{2} M \eta^{1}+\eta^{3}+M \eta_{, a}^{1}+2 \eta_{, a}^{3}\right]+M(f+g)_{, \phi} \eta^{4}-f M e^{-b / 2} \eta_{, r}^{3}+\frac{1}{2} g_{, \phi} M^{2} e^{-b / 2} \eta_{, r}^{4}+e^{-a / 2} G_{, a}=0, \\
& (f+g)\left[M \eta_{, b}^{1}+2 \eta_{, b}^{3}\right]+e^{-a / 2} G_{, b}=0, \\
& (f+g)\left(\eta^{1}+M \eta_{, M}^{1}+2 \eta_{, M}^{3}\right)+2(f+g)_{, \phi} \eta^{4}-f M e^{-b / 2} \eta_{, r}^{1}-f e^{-b / 2} \eta_{, r}^{3}+2 g_{, \phi} M e^{-b / 2} \eta_{, r}^{4}+e^{-a / 2} G_{, M}=0, \\
& (f+g)\left[M \eta_{, \phi}^{1}+2 \eta_{, \phi}^{3}\right]+g_{\phi} e^{-b / 2}\left(\frac{1}{2} M^{2} \eta_{, r}^{1}+2 M \eta_{, r}^{3}\right)+M^{2} e^{-b / 2} \eta_{, r}^{4}+e^{-a / 2} G_{, \phi}=0, \\
& \left(M^{2} V(\phi)-f\right)\left[\frac{1}{2}\left(\eta^{1}+\eta^{2}\right)-\xi_{, r}\right]+2 M V(\phi) \eta^{3}+\left(M^{2} V(\phi)_{, \phi}-f_{, \phi}\right) \eta^{4} \\
& +e^{-b / 2}(f+g)\left(M \eta_{, r}^{1}+2 \eta_{, r}^{3}\right)+e^{-(a+b) / 2} G_{, r}=0 \text {. }
\end{aligned}
$$

It is explicitly seen from the above equations that $\xi=\xi(r)$, and a trivial solution is $\xi=$ const, $\eta^{i}=0$ and $G=$ const. This trivial solution means that $\mathbf{X}_{1}=$ $\partial_{r}$ is a Noether symmetry for any form of the $f(\phi)$, $g(\phi)$ and $V(\phi)$. The corresponding Noether constant of $\mathbf{X}_{1}$ is $I=-E_{\mathcal{L}}$ which vanishes due to the Eq. (34).

Case (i): $V(\phi)=0$ (vanishing potential).

Relevant subcases:

(i.a). Take $f(\phi)=f_{0}-\zeta \phi^{2}$ and $g(\phi)=-\chi \phi^{2}$, where $f_{0}, \zeta$ and $\chi$ are constants.

(i.a.1) If $\chi=0$, then we have teleparallel dark energy (nonminimally coupled to $T$ ).

(i.a.2) If $\chi=-\zeta$, then it means that dark energy is nonminimally coupled to the Ricci scalar.

(i.a.3) If $\zeta=0$, then it gives dark energy nonminimally coupled to the boundary term.

(i.a.4) The condition $\zeta=\chi=0$ gives rise to quintessence models.

(i.b). If $f(\phi)=f_{0}-\zeta \phi$ and $g(\phi)=-\chi \phi$, then the possibilities are

(i.b.1) $\chi=0$, (i.b.2) $\chi=-\zeta$ [Brans-Dicke theory with $w(\phi)=1]$, (i.b.3) $\zeta=0$, and (i.b.4). The condition $\zeta=\chi=0$ gives rise to quintessence models the same as subcase (i.a.4).

The Noether symmetries for the Lagrangian density (32) are obtained as

$$
\begin{aligned}
\xi & =c_{1}, \quad \eta^{1}=-2 c_{2}, \quad \eta^{2}=2 c_{2}, \\
\eta^{3} & =c_{2} M, \quad \eta^{4}=0, \quad G=c_{3},
\end{aligned}
$$

for the subcases (i.a.1), (i.a.2), (i.a.3), (i.b.1) and (i.b.2) which yields that

$$
\mathbf{X}_{1}=\partial_{r}, \quad \mathbf{X}_{2}=-\partial_{a}+\partial_{b}+\frac{1}{2} M \partial_{M}
$$

The corresponding first integrals of the above Noether symmetries are

$$
I_{1}=-E_{\mathcal{L}}, \quad I_{2}=\frac{1}{2} f(\phi) M^{2} e^{(a-b) / 2} a^{\prime},
$$

where $I_{1}$ vanishes due to the $E_{\mathcal{L}}=0$. The second of the above first integrals gives $\left(e^{a / 2}\right)^{\prime}=\alpha e^{b / 2} /\left[f(\phi) M^{2}\right]$, where $\alpha=I_{2}$. Thus the equation $E_{\mathcal{L}}=0$ takes the form 


$$
u^{\prime 2}=\frac{\alpha^{2}}{\left(f_{0}-\zeta \phi^{2}\right)^{3} M^{2}}\left[2 P \frac{u^{\prime}}{u}+Q\right],
$$

where $u$ is defined as $u \equiv e^{a / 2} ; P$ and $Q$ are defined by

$$
\begin{aligned}
& P=\chi \phi \phi^{\prime}+\left(f_{0}-\zeta \phi^{2}\right) \frac{M^{\prime}}{M}, \\
& Q=\frac{M^{\prime}}{M}\left(P+3 \chi \phi \phi^{\prime}\right)-\frac{\phi^{\prime 2}}{2} .
\end{aligned}
$$

Equation (49) is a nonlinear differential equation for $u$, and it could be solved under some assumptions.

In subcase (i.b.3), i.e. $f(\phi)=f_{0}$ and $g(\phi)=-\chi \phi$, we have three Noether symmetries $\mathbf{X}_{1}, \mathbf{X}_{2}$ and $\mathbf{X}_{3}=\partial_{\phi}$ with $G=2 \chi M e^{a / 2}$. Thus we found the first integrals in this case as $I_{1}=-E_{\mathcal{L}}=0, I_{2}=\frac{1}{2} f_{0} M^{2} e^{(a-b) / 2} a^{\prime}$ and $I_{3}=$ $-M^{2} e^{(a-b) / 2} \phi^{\prime}-2 \chi M e^{a / 2}$.

(i.c). When $g(\phi)=-f(\phi)$ and $f(\phi)=f_{0}$ (const.), the components of Noether symmetry generator $\mathbf{X}$ are

$$
\begin{aligned}
\xi & =c_{1}+c_{2} r, \quad \eta^{1}=2 c_{2}(1+\ln M)-2 c_{3} \phi-2 c_{4}, \\
\eta^{2} & =-2 c_{2} \ln M+2 c_{3} \phi+2 c_{4}, \\
\eta^{3} & =M\left(-c_{2} \ln M+c_{3} \phi+c_{4}\right), \\
\eta^{4} & =c_{3} f_{0} a+c_{5}, G=c_{6} .
\end{aligned}
$$

Then the Noether symmetries are $\mathbf{X}_{1}, \mathbf{X}_{2}$ given in (47) and

$$
\begin{aligned}
& \mathbf{X}_{3}=\partial_{\phi}, \quad \mathbf{X}_{4}=\phi \mathbf{X}_{2}+\frac{1}{2} f_{0} a \mathbf{X}_{3}, \\
& \mathbf{X}_{5}=\frac{r}{2} \mathbf{X}_{1}-(\ln M) \mathbf{X}_{2}+\partial_{a}
\end{aligned}
$$

with the nonvanishing Lie brackets

$$
\left[\mathbf{X}_{1}, \mathbf{X}_{5}\right]=\frac{1}{2} \mathbf{X}_{1}, \quad\left[\mathbf{X}_{2}, \mathbf{X}_{4}\right]=-\frac{1}{2} f_{0} \mathbf{X}_{3}, \quad\left[\mathbf{X}_{2}, \mathbf{X}_{5}\right]=-\frac{1}{2} \mathbf{X}_{2}
$$

$\left[\mathbf{X}_{3}, \mathbf{X}_{4}\right]=\mathbf{X}_{2}, \quad\left[\mathbf{X}_{4}, \mathbf{X}_{5}\right]=-\frac{1}{2} \phi \mathbf{X}_{2}-\frac{1}{2} f_{0}(1+\ln M) \mathbf{X}_{3}$.

The corresponding Noether constants are

$I_{1}=-E_{\mathcal{L}}, \quad I_{2}=\frac{f_{0}}{2} M^{2} e^{(a-b) / 2} a^{\prime}, \quad I_{3}=-M^{2} e^{(a-b) / 2} \phi^{\prime}$,

$I_{4}=I_{2} \phi+\frac{1}{2} f_{0} I_{3} a, \quad I_{5}=-I_{2}(\ln M)+f_{0} e^{(a-b) / 2} M M^{\prime}$, where $I_{1}=0$ because of the fact $E_{\mathcal{L}}=0$, which yields

$$
e^{b(r)}=M M^{\prime} a^{\prime}+M^{\prime 2}-\frac{M^{2}}{2 f_{0}} \phi^{\prime 2} .
$$

Using the first integral $I_{2}=\frac{f_{0}}{2} M^{2} e^{(a-b) / 2} a^{\prime}$, we have

$$
e^{(b-a) / 2}=\frac{f_{0} M^{2}}{I_{2}} \frac{u^{\prime}}{u}
$$

where $I_{2} \neq 0$ and $u \equiv e^{a / 2}$. Then, putting the above term into the first integral $I_{5}=-I_{2}(\ln M)+f_{0} e^{(a-b) / 2} M M^{\prime}$, it becomes

$$
\frac{u^{\prime}}{u}=\frac{I_{2} \frac{M^{\prime}}{M}}{I_{2} \ln M+I_{5}},
$$

which has the solution $\ln u=I_{2} \ln M+I_{5}$, that is, $e^{a(r)}=$ $e^{2 I_{5}} M^{2 I_{2}}$ or $a(r)=2\left(I_{2} \ln M+I_{5}\right)$. Taking $I_{5}=-I_{2} \ln M_{0}$, one can write that

$$
e^{a(r)}=\left(\frac{M}{M_{0}}\right)^{2 I_{2}},
$$

where $M\left(r_{0}\right)=M_{0}$. Thus, together with the first integral $I_{3}=-M^{2} e^{(a-b) / 2} \phi^{\prime}$, Eq. (57) yields

$$
e^{b(r)}=\frac{\left(2 I_{2}+1\right) M^{\prime 2}}{1-\ell\left(M / M_{0}\right)^{-2\left(I_{2}+1\right)}},
$$

where $\ell=-I_{3}^{2} /\left(2 f_{0} M_{0}^{2}\right)$. The shape function of the wormhole becomes

$$
\beta(r)=r\left(1-\frac{\left[1-\ell\left(M / M_{0}\right)^{-2\left(I_{2}+1\right)}\right]}{\left(2 I_{2}+1\right) M^{\prime 2}}\right),
$$

where $f_{0} \neq 0, M^{\prime} \neq 0$ and $I_{2} \neq-1 / 2$. The function $M(r)$ is independent of $\theta$ and $\phi$ because of isotropy, and it must have the limit $M(r) \sim r$ as $r \rightarrow 0$. The form of the function $M(r)$ can be determined by use of the geodesic deviation equation (see page 50 of Ref. [32]), which gives that the solutions for $M(r)$ with the appropriate limit behavior $M(r) \sim r$ as $r \rightarrow 0$ are $M(r)=\epsilon^{-1} \sin (\epsilon r)$ if $k=1, r$ if $k=0$ and $\epsilon^{-1} \sinh (\epsilon r)$ if $k=-1$, where $k$ is the curvature parameter and $\epsilon$ is a dimensional constant. In the limit case $M(r)=r$, the metric coefficients, the shape function and the scalar field take the form

$$
\begin{array}{r}
e^{a(r)}=\left(\frac{r}{r_{0}}\right)^{2 I_{2}}, \quad e^{b(r)}=\frac{\left(2 I_{2}+1\right)}{1-\ell\left(\frac{r}{r_{0}}\right)^{-2\left(I_{2}+1\right)},} \\
\beta(r)=r\left[1-\frac{1}{\left(2 I_{2}+1\right)}+\frac{\ell}{\left(2 I_{2}+1\right)}\left(\frac{r}{r_{0}}\right)^{-2\left(I_{2}+1\right)}\right],
\end{array}
$$



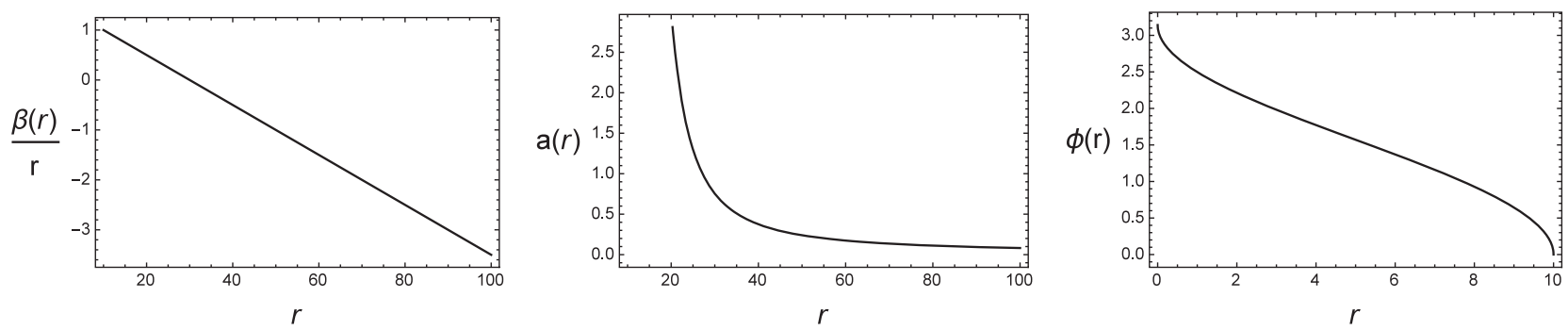

FIG. 1. Shape function divided by $r$, redshift function and scalar field versus the radial coordinate for $\ell=1, I_{2}=-1.5, f_{0}=-1$ and $r_{0}=10$

$\phi(r)=-\frac{1}{\left(I_{2}+1\right)} \sqrt{\frac{2 I_{2}+1}{2 f_{0}}} \arctan \left[\sqrt{\frac{1}{\ell}\left(\frac{r}{r_{0}}\right)^{2\left(I_{2}+1\right)}-1}\right.$.

The metric coefficients (63) and the shape function (64) are very similar to the wormhole solution found by Cataldo et al. [33] under the assumption of isotropic pressure. It can be noted that in this solution we must set $\ell=1$ to satisfy the wormhole condition at the throat $\beta\left(r=r_{0}\right)=r_{0}$. Figures 1 and 2 show the behavior of this solution for $\beta(r) / r, a(r)$ and $\phi(r)$ for different values of the parameters $I_{2}, r_{0}$ and $f_{0}$. In order to have a real and positive scalar field, the parameters must satisfy $I_{2}<-1$ and $f_{0}<0$. Moreover, the scalar field must lie outside the wormhole in the region $\left[0, r_{0}\right]$; otherwise it will become negative or complex. We can see from the pictures that the wormhole obeys the asymptotic flatness in both cases since as $r \rightarrow \infty$, a de Sitter spacetime is recovered.

For $M(r)=\epsilon^{-1} \sin (\epsilon r)$, we find that

$e^{a(r)}=\left(\frac{\sin (\epsilon r)}{\sin \left(\epsilon r_{0}\right)}\right)^{2 I_{2}}, \quad e^{b(r)}=\frac{\left(2 I_{2}+1\right) \cos ^{2}(\epsilon r)}{1-\ell\left(\frac{\sin (\epsilon r)}{\sin \left(\epsilon r_{0}\right)}\right)^{-2\left(I_{2}+1\right)}}$,

$\beta(r)=r\left[1-\frac{1}{\left(2 I_{2}+1\right) \cos ^{2}(\epsilon r)}\left\{1-\left(\ell \frac{\sin \left(\epsilon r_{0}\right)}{\sin (\epsilon r)}\right)^{2\left(I_{2}+1\right)}\right\}\right]$, which is a new wormhole solution as far as we know. One can find a similar solution for $M(r)=\epsilon^{-1} \sinh (\epsilon r)$. If $g(\phi)=0$, which corresponds to the subcases (i.a.1) and (i.b.1), then the Noether symmetries are $\mathbf{X}_{1}$ and $\mathbf{X}_{2}$ given in (47) for arbitrary $f(\phi)$.

(i.d). If $g(\phi)=0$ and $f(\phi)=\left(f_{0}+f_{1} \phi\right)^{2}$, where $f_{1} \neq 0$, the solution of Noether symmetry equations (45) is

$$
\xi=c_{1}, \quad \eta^{1}=-2 c_{2}-2 c_{3}\left(1+\frac{1}{M}+\ln M\right),
$$

$\eta^{2}=2 c_{2}+2 c_{3}\left(\frac{1}{M}+\ln M\right)$

$\eta^{3}=c_{2} M+c_{3}(M \ln M+1), \quad \eta^{4}=\frac{c_{3}}{2 f_{1}}\left(f_{0}+f_{1} \phi\right)$,

$G=c_{4}$,

that is, the $\mathbf{X}_{1}, \mathbf{X}_{2}$ by (47) and

$\mathbf{X}_{3}=\left(\ln M+\frac{1}{M}\right) \mathbf{X}_{2}-\partial_{a}+\frac{1}{4 f_{1}}\left(f_{0}+f_{1} \phi\right) \partial_{\phi}$

are Noether symmetries. Here there is only the nonvanishing Lie bracket due to the $\mathbf{X}_{1}, \mathbf{X}_{2}$ and $\mathbf{X}_{3}$ as

$$
\left[\mathbf{X}_{2}, \mathbf{X}_{3}\right]=\frac{1}{2}\left(1-\frac{1}{M}\right) \mathbf{X}_{2}
$$

Here the first integrals for $\mathbf{X}_{1}, \mathbf{X}_{2}$ and $\mathbf{X}_{3}$ are $I_{1}=-E_{\mathcal{L}}=$ $0, I_{2}=\frac{1}{2}\left(f_{0}+f_{1} \phi^{2}\right) M^{2} e^{(a-b) / 2} a^{\prime}$ and
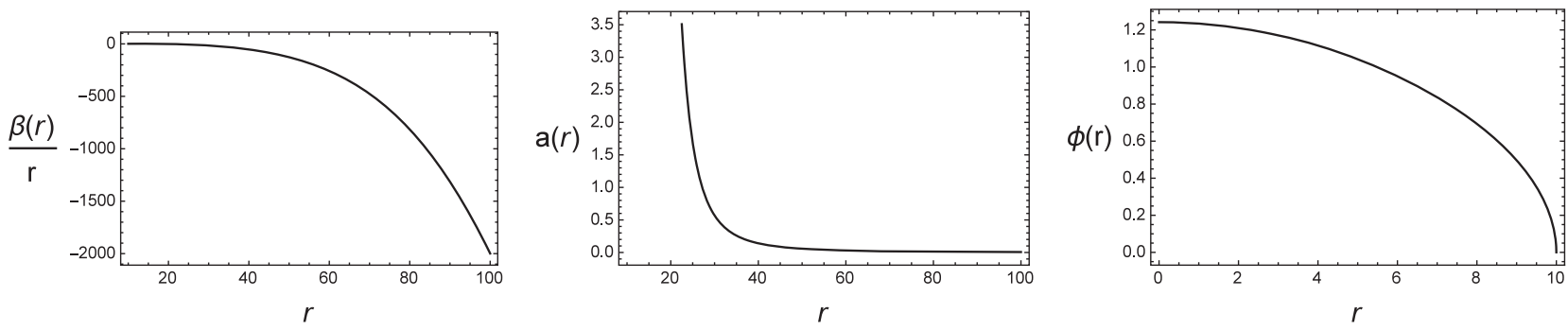

FIG. 2. Shape function divided by $r$, redshift function and scalar field versus the radial coordinate for $\ell=1, I_{2}=-3, f_{0}=-1$ and $r_{0}=10$ 


$$
\begin{aligned}
I_{3}= & I_{2}\left(\frac{1}{M}+\ln M\right)-\left(f_{0}+f_{1} \phi\right)^{2}\left(e^{(a-b) / 2} M M^{\prime}-e^{a / 2} M\right) \\
& -\frac{1}{4 f_{1}}\left(f_{0}+f_{1} \phi\right) M^{2} e^{(a-b) / 2} \phi^{\prime} .
\end{aligned}
$$

If $g(\phi)=0$ and $f(\phi)=f_{0}=$ const, which corresponds to the subcase (i.a.4) or (i.b.4), then the components of Noether symmetry generator $\mathbf{X}$ are the same as (51), but the gauge function $G$ is obtained by $G=-2 c_{2} f_{0} M e^{a / 2}+c_{6}$. Therefore in this case the Noether constant $I_{5}$ related to $\mathbf{X}_{5}$ has the form

$$
I_{5}=-I_{2}(\ln M)+f_{0} e^{(a-b) / 2} M M^{\prime}+f_{0} M e^{a / 2} .
$$

Case (ii): $V(\phi)=V_{0}$ (constant potential).

When $g(\phi)=-f(\phi)$ and $f(\phi)=f_{0}$ or $g(\phi)=0$ and $f(\phi)=f_{0}$ (constant), we have only two Noether symmetries $\mathbf{X}_{1}=\partial_{r}$ and $\mathbf{X}_{2}=\partial_{\phi}$ which gives rise to the Noether constants $I_{1}=-E_{\mathcal{L}} \equiv 0$ and $I_{2}=-M^{2} e^{(a-b) / 2} \phi^{\prime}$. Thus, the relation $E_{\mathcal{L}}=0$ gives

$$
e^{b(r)}=\frac{e^{a}\left(M M^{\prime} a^{\prime}+M^{\prime 2}\right)}{e^{a}\left(1-\frac{V_{0} M^{2}}{f_{0}}\right)+\frac{I_{2}^{2}}{2 f_{0} M^{2}}} .
$$

For $M(r)=r$, it follows that

$$
e^{b(r)}=\frac{e^{a}\left(r a^{\prime}+1\right)}{e^{a}\left(1-\frac{V_{0} r^{2}}{f_{0}}\right)+\frac{I_{2}^{2}}{2 f_{0} r^{2}}},
$$

which yields the shape function of the wormhole as

$$
\beta(r)=\frac{r\left[e^{a}\left(r a^{\prime}+\frac{V_{0} r^{2}}{f_{0}}\right)-\frac{I_{2}^{2}}{2 f_{0} r^{2}}\right]}{e^{a}\left(r a^{\prime}+1\right)} .
$$

Considering the specific redshift function given by $a(r)=0$ [31], and inserting this condition into Eq. (75) the shape function reduces to the form

$$
\beta(r)=\frac{r}{f_{0}}\left(V_{0} r^{2}-\frac{I_{2}^{2}}{2 r^{2}}\right) .
$$

Now, we take into account the Noether first integral $I_{2}=-r^{2} e^{(a-b) / 2} \phi^{\prime}$, and find that

$$
\begin{aligned}
\phi(r)= & I_{2} \sqrt{\frac{f_{0}}{2}} \ln \left[2 \left(\sqrt{\frac{2}{f_{0}}} \frac{I_{2}}{r} \sqrt{1-\frac{V_{0} r^{2}}{f_{0}}+\frac{I_{2}^{2}}{2 f_{0} r^{2}}}\right.\right. \\
& \left.\left.+\frac{I_{2}^{2}}{f_{0} r^{2}}+1\right)\right]+\phi_{0},
\end{aligned}
$$

where $\phi_{0}$ is an integration constant. For $V(\phi)=V_{0} \phi^{n}$, where $n=1,2, \ldots$, there is only one Noether symmetry $\mathbf{X}_{1}=\partial_{r}$, which is a trivial solution and corresponds to the first integral $I=-E_{\mathcal{L}}=0$.

\section{DISCUSSION AND CONCLUSIONS}

Teleparallel theories and their extensions have gained a lot of attention in recent years as alternative gravitation frameworks. Additionally, in the literature, these theories have been studied by their couplings, both minimal and nonminimal, with torsion. Our choice of action generalizes and extends most of the earlier models of teleparallel gravity by assuming an additional coupling between the scalar field and a boundary term $B$ which is related to the divergence of torsion. Moreover, this new coupling allows us to formulate a theory that, under some constraints, allows us to construct quintessence, teleparallel dark energy and a nonminimally coupled scalar field to the Ricci scalar theories. The latter has been used in the literature to study spherically symmetric configurations as wormholes (see Ref. [24]) obtaining the existence of traversable wormholes supported by nonminimally coupled scalars.

Here we derived new wormhole solutions according to the Morris and Thorne paradigm. In this connection, we adopt the Noether symmetry approach solving the governing equations to obtain symmetry generators, the gauge function and the metric coefficients of the wormhole geometry. To perform this analysis, we focused on two forms of potential function, i.e. $V=0$ and $V=$ constant. In the former case, we obtained five symmetry generators while in the latter case, only two. For the vanishing potential, we explicitly found two different wormhole solutions in the case $f(\phi)=-g(\phi)=$ constant, i.e., within a quintessence model. The first wormhole solution is similar to that found by Cataldo et al. [33] under the assumption of isotropic pressure, whereas the second wormhole solution is, in our knowledge, a new wormhole solution.

It can be seen that these solutions respect the asymptotic flatness as $r \rightarrow \infty$. In the case where a constant potential is assumed, we also found an explicit wormhole solution for the quitessence case $(g=0$ and $f=$ constant). For a power-law form of potential, only one generator exists which corresponds to a trivial solution.

It might be interesting to perform a similar analysis by assuming a more general action as, for example, to include also coupling between the scalar field and the teleparallel torsion Gauss Bonnet term $T_{G}$ or a Gauss Bonnet boundary term $B_{G}$ (see for example Ref. [12]). These will be the arguments of future work.

\section{ACKNOWLEDGMENTS}

S. C. acknowledges the support of Istituto Nazionale di Fisica Nucleare, Sezione di Napoli, Italy, iniziative specifiche TEONGRAV and QGSKY.S. B. and S.C. also acknowledge the European Cooperation in Science and Technology (COST) action Grant No. CA15117 (CANTATA), supported by COST. S. B. is supported by the Comisión Nacional de Investigación Científica y Tecnológica (Becas Chile Grant No. 72150066) 
[1] M. S. Morris and K. S. Thorne, Am. J. Phys. 56, 395 (1988).

[2] L. Flamm, Phys. Z. 17, 448 (1916).

[3] M. Jamil, M. U. Farooq, and M. A. Rashid, Eur. Phys. J. C 59, 907 (2009); M. Jamil, P. K. F. Kuhfittig, F. Rahaman, and Sk. A. Rakib, Eur. Phys. J. C 67, 513 (2010).

[4] R. Myrzakulov, Gen. Relativ. Gravit. 44, 3059 (2012).

[5] K. Bamba, S. Capozziello, S. Nojiri, and S. D. Odintsov, Astrophys. Space Sci. 342, 155 (2012).

[6] S. Nojiri and S. D. Odintsov, Int. J. Geom. Methods Mod. Phys. 04, 115 (2007).

[7] S. Capozziello and M. De Laurentis, Phys. Rep. 509, 167 (2011).

[8] S. Nojiri and S. D. Odintsov, Phys. Rep. 505, 59 (2011).

[9] Y.-F. Cai, S. Capozziello, M. De Laurentis, and E. N. Saridakis, Rep. Prog. Phys. 79, 106901 (2016).

[10] M. Jamil, D. Momeni, and R. Myrzakulov, Eur. Phys. J. C 72, 1959 (2012); 72, 2122 (2012).

[11] S. Bahamonde, C. G. Böhmer, and M. Wright, Phys. Rev. D 92, 104042 (2015).

[12] S. Bahamonde and C. G. Boehmer, arXiv:1606.05557.

[13] S. Capozziello, M. De Laurentis, and R. Myrzakulov, Int. J. Geom. Methods Mod. Phys. 12, 1550095 (2015).

[14] M. Jamil, D. Momeni, and R. Myrzakulov, Eur. Phys. J. C 72, 2075 (2012); 72, 2137 (2012); Int. J. Theor. Phys. 54, 1098 (2015); A. Aslam, M. Jamil, D. Momeni, and R. Myrzakulov, Can. J. Phys. 91, 93 (2013).

[15] S. Capozziello, R. De Ritis, C. Rubano, and P. Scudellaro, Nuovo Cimento Soc. Ital. Fis. 19, 1 (1996).

[16] S. Basilakos, S. Capozziello, M. De Laurentis, A. Paliathanasis, and M. Tsamparlis, Phys. Rev. D 88, 103526 (2013); S. Capozziello and R. de Ritis, Classical Quantum Gravity 24, 2153 (2007).

[17] A. Paliathanasis, S. Basilakos, E. N. Saridakis, S. Capozziello, K. Atazadeh, F. Darabi, and M. Tsamparlis, Phys. Rev. D 89, 104042 (2014); I. Hussain, M. Jamil, and F. M. Mahomed, Astrophys. Space Sci. 337, 373 (2012); M. Jamil, F. M. Mahomed, and D. Momeni, Phys. Lett. B 702, 315 (2011).

[18] A. Aslam, M. Jamil, and R. Myrzakulov, Phys. Scr. 88, 025003 (2013).
[19] Y. Kucukakca, Eur. Phys. J. C 73, 2327 (2013).

[20] Y. Kucukakca, Eur. Phys. J. C 74, 3086 (2014).

[21] A. K. Ahmed, M. Azreg-Ainou, S. Bahamonde, S. Capozziello, and M. Jamil, Eur. Phys. J. C 76, 269 (2016).

[22] S. Bahamonde and M. Wright, Phys. Rev. D 92, 084034 (2015).

[23] M. Zubair and S. Bahamonde, arXiv:1604.02996.

[24] C. Barcelo and M. Visser, Classical Quantum Gravity 17, 3843 (2000).

[25] S. V. Sushkov and S. W. Kim, Classical Quantum Gravity 19, 4909 (2002).

[26] K. A. Bronnikov and S. V. Grinyok, Gravitation Cosmol. 7, 297 (2001).

[27] K. A. Bronnikov and S. V. Grinyok, Gravitation Cosmol. 11, 75 (2005).

[28] E. F. Eiroa, M. G. Richarte, and C. Simeone, Phys. Lett. A 373, 1 (2008); 373, 2399(E) (2009); A. G. Agnese and M. La Camera, Phys. Rev. D 51, 2011 (1995); K. K. Nandi, A. Islam, and J. Evans, Phys. Rev. D 55, 2497 (1997); F. He and S.-W. Kim, Phys. Rev. D 65, 084022 (2002); A. Bhadra and K. Sarkar, Mod. Phys. Lett. A 20, 1831 (2005); K. A. Bronnikov and A. A. Starobinsky, JETP Lett. 85, 1 (2007); K. A. Bronnikov and A. A. Starobinsky, Mod. Phys. Lett. A 24, 1559 (2009); K. A. Bronnikov, M. V. Skvortsova, and A. A. Starobinsky, Gravitation Cosmol. 16, 216 (2010).

[29] D. F. Torres, Phys. Rev. D 66, 043522 (2002); L. Amendola, Phys. Rev. D 62, 043511 (2000); V. Faraoni, Int. J. Mod. Phys. D11, 471 (2002).

[30] B. Boisseau, G. Esposito-Farese, D. Polarski, and A. A. Starobinsky, Phys. Rev. Lett. 85, 2236 (2000); F. Perrotta, C. Baccigalupi, and S. Matarrese, Phys. Rev. D 61, 023507 (1999); C. Q. Geng, C. C. Lee, E. N. Saridakis, and Y. P. Wu, Phys. Lett. B 704, 384 (2011).

[31] C. G. Boehmer, T. Harko, and F. S. N. Lobo, Phys. Rev. D 85, 044033 (2012).

[32] G. F. R. Ellis, R. Maartens, and M. A. H. MacCallum, Relativistic Cosmology (Cambridge University Press, Cambridge, 2012).

[33] M. Cataldo, L. Liempi, and P. Rodriguez, Phys. Lett. B 757, 130 (2016). 